\title{
Evaluation of the Panbio Leptospira IgM ELISA among Outpatients Attending Primary Care in Southeast Asia
}

\author{
Sandhya Dhawan, ${ }^{1}$ Thomas Althaus, ${ }^{1,2}$ Yoel Lubell, ${ }^{1,2}$ Duangjai Suwancharoen, ${ }^{3}$ and Stuart D. Blacksell ${ }^{1,2,4 \star}$ \\ ${ }^{1}$ Mahidol-Oxford Tropical Research Medicine Unit, Faculty of Tropical Medicine, Mahidol University, Bangkok, Thailand; ${ }^{2}$ Nuffield Department of \\ Medicine, Nuffield Department of Medicine Research Building, Centre for Tropical Medicine and Global Health, University of Oxford, Oxford, \\ United Kingdom; ${ }^{3}$ Department of Livestock Development, National Institute of Animal Health, Ministry of Agriculture and Cooperatives, \\ Bangkok, Thailand; ${ }^{4}$ Lao-Oxford-Mahosot Hospital-Wellcome Trust Research Unit, Microbiology Laboratory, Mahosot Hospital, Vientiane, Lao \\ People's Democratic Republic
}

\begin{abstract}
Despite estimates suggesting Leptospira spp. being endemic in Southeast Asia, evidence remains limited. Diagnostic accuracy evaluations based on Leptospira ELISA mainly rely on hospitalized and severe patients; therefore, studies measuring the pathogen burden may be inaccurate in the community. We evaluated the Panbio Leptospira ELISA IgM among 656 febrile outpatients attending primary care in Chiangrai, Thailand, and Hlaing Tha Yar, Yangon, Myanmar. ELISA demonstrated limited diagnostic accuracy for the detection of acute leptospiral infection using the manufacturer recommended cutoff, with a sensitivity of $71.4 \%$ and specificity of $36.4 \%$, and an area under the receiver operator characteristic curve value of 0.65 (95\% Cl: $0.41-0.89)$, compared with our reference test, the PCR assay. ELISA also performed poorly as a screening tool for detecting recent exposure to Leptospira spp. compared with the "gold-standard" microscopic agglutination test, with a specificity of $42.7 \%$. We conclude that the utility of the Leptospira IgM ELISA for both serodiagnosis and seroprevalence is limited in our setting.
\end{abstract}

\section{INTRODUCTION}

Leptospirosis is a widespread but neglected zoonotic disease caused by a bacterium of the genus Leptospira. ${ }^{1,2}$ Leptospirosis contributes to approximately 48,000 annual deaths globally, ${ }^{3-5}$ with 500,000 cases estimated worldwide, ${ }^{6}$ and remains a major public health concern in tropical and subtropical regions. ${ }^{7}$ Agriculturers and animal farmers are at a higher risk, but also in case of poor sanitation, ${ }^{2}$ with evidence of pathogenic Leptospira recently reported in urban poor areas. ${ }^{2,7-10}$ The endemicity of leptospirosis is primarily localized to Southeast Asia, where the burden of the disease is concentrated. ${ }^{11,12}$ In Thailand, it is a major public health concern with several outbreaks reported annually, ${ }^{11,13}$ and a background prevalence persisting at five cases per 100,000 population per year according to the CDC. ${ }^{13}$ Moreover, a recent study at the Thai-Myanmar border established leptospirosis as the second most common cause of undifferentiated febrile illness in this region, ${ }^{14}$ suggesting that the disease may be endemic in Myanmar as well.

However, underdiagnosis and underreporting of leptospirosis remain considerable problems. Clinical presentation is not specific, overlapping with common tropical pathogens such as dengue virus or other hemorrhagic febrile illnesses. ${ }^{6}$ Furthermore, Leptospira spp. is inconsistently integrated in Southeast Asian national surveillance, without standardized laboratory data collection nor reporting systems, explaining gaps in the disease true burden estimate. ${ }^{12}$

The serological "gold standard" for identifying pathogenic Leptospira is the microscopic agglutination test (MAT); however, it requires significant technical expertise and the maintenance of viable Leptospira serovars. Microscopic agglutination test is often not beneficial for acute patient management, mainly because it relies on immunoglobulins which react 5 days after the exposure. This is illustrated by a sensitivity of approximately

\footnotetext{
*Address correspondence to Stuart D. Blacksell, Mahidol-Oxford Tropical Medicine Research Unit, Faculty of Tropical Medicine, Mahidol University, Bangkok 10400, Thailand. E-mail: stuart@
} tropmedres.ac
$50 \% .^{7,15,16}$ On the other hand, MAT is serovar specific, with specificity ranging from 94 to $97 \% .^{16-19}$ Microscopic agglutination test is also considered the "gold standard" for seroprevalence studies and epidemiological surveys, where exposure to Leptospira spp. is measured instead of overt disease. ${ }^{20,21}$ Given the high specificity of MAT, estimates of exposure are likely to be very accurate.

Molecular methods such as the PCR may be of greater performance for the diagnosis of acute leptospirosis, by improving the Leptospira spp. early phase detection with a sensitivity ranging from 51 to $100 \%{ }^{2,22,23}$ Based on PCR, a multicountry study based in Africa reported a sensitivity near $100 \%$ with $95 \%$ specificity using the TaqMan Array Card (TAC) assay, compared with MAT and single-plex PCR-confirmed samples, ${ }^{24}$ hence indisputable evidence of acute infection. ${ }^{25}$

An alternative diagnostic strategy relies on the ELISA, which is widely used for detecting Leptospira spp. antibodies. ELISA IgM antibodies are detectable 5 days after exposure and only persist for a few months, suggesting a potential application both in acute diagnosis and recent exposure to Leptospira spp., whereas the procedure is simple to perform with results available in around 2 hours. ${ }^{26}$

For serodiagnosis purpose, diagnostic accuracy evaluations have shown inconsistent sensitivity and specificity. ${ }^{5,23,27,28}$ This lack of consistency may be explained by the specific interaction between the background immunity of a given population and the exposure chronicity. ${ }^{15,29}$ In Southeast Asia, recent studies have confirmed broad variations in diagnostic accuracy, with a sensitivity ranging from $36.0 \%$ to $60.9 \%$ and a specificity ranging from $41 \%$ to $98.0 \%{ }^{15,29-31}$ These variations also reflect imperfect reference methods, such as the MAT or blood culture: performances of which fluctuate depending on symptom onset and diagnostic cutoff. ${ }^{27}$ Furthermore, most samples included in ELISA diagnostic evaluations originate from severe hospitalized patients, implying particular immunological profiles and limited application to leptospirosis with a mild presentation. A single study recruited outpatients in southern Vietnam, and attributed the diagnosis of acute leptospirosis without any reference method. ${ }^{30}$ Therefore, the utility of 
commercial lgM ELISA kits for serodiagnosis of Leptospira spp. remains unclear, particularly among non-severe outpatients attending the primary levels of care.

ELISA has also been used for seroprevalence purposes, with the objective to screen for exposure to Leptospira spp. ${ }^{21,32-37}$ Alarmingly, a single study evaluated the ELISA diagnostic accuracy for measuring such exposure in Southeast Asia, using MAT as a reference test and reporting $24.3 \%$ sensitivity and $93.5 \%$ specificity. ${ }^{20}$ In this Malaysian study, samples were exclusively taken from a particular subpopulation of market workers and food handlers, whereas children were excluded from the evaluation. Whether IgM ELISA could be an accurate screening tool for recent exposure to pathogenic Leptospira in the general population attending primary care is unknown.

In this study, we report the diagnostic accuracy of the commercial Leptospira IgM ELISA (Panbio Pty., Ltd., Queensland, Australia) compared with single-plex PCR and TAC assays among both children and adults attending primary care in Chiang Rai, Thailand, and Yangon, Myanmar. We also measure exposure to Leptospira spp. using the MAT, and calculate the corresponding IgM ELISA specificity as a screening tool for recent exposure.

\section{METHODS}

Study sites. Samples were analyzed from the CRP Study, which was conducted in 10 primary care centers, of which six were located in Chiang Rai Province, northern Thailand and four in Hlaing Tha Yar, Yangon, Myanmar. ${ }^{38}$ Chiang Rai Province is located in the north of Thailand and borders Myanmar and Lao People's Democratic Republic (PDR). The sites included in this study were within a 30-km radius from Chiang Rai city center, and included rural, peri-urban, mountainous, and plateau areas. Hlaing Tha Yar is a peri-urban slum in the west of Yangon Myanmar. All study sites are defined by their tropical climate, with a population composed by ethnic minorities.

Patient details. Patients ( $\geq 1$ year) were recruited between June 2016 and August 2017. Inclusion criterion was a documented fever (tympanic temperature $>37.5^{\circ} \mathrm{C}$ ) or a complaint of acute fever (< 14 days). Patients were excluded if they presented with symptoms requiring hospital referral such as impaired consciousness, inability to take oral medication, etc. On enrollment, the patients' demographic information was collected, and a routine clinical examination was performed.

Leptospira testing. Of 2,392 primary care children and adults recruited in the original trial, 799 were randomized in a control group for whom various samples were collected. ${ }^{38}$ From these 799 outpatients, 740 had a blood sample collected. Samples were stored onsite at $-80^{\circ} \mathrm{C}$ and then shipped to the Mahidol-Oxford Tropical Medicine Research Unit and National Institute of Animal Health for microbiological investigations. A commercial ELISA (Panbio Pty., Ltd.) was used for the detection of IgM antibodies against Leptospira spp. The Manufacturer's specifications were followed with Panbio units of $\geq 11.0$ considered positive. Positive Leptospira IgM ELISA samples were then tested by MAT, with titers of $\geq 1: 100$ used to classify past exposure to leptospirosis. ${ }^{20,21,39-41}$ TaqMan Array Card and single-plex PCR assays were performed on 601 blood samples where sufficient plasma volumes were able to be extracted. The TAC assay targeted all pathogenic serovars of the Leptospira genus, whereas the single-plex PCR used real-time methodology to target the rss gene ${ }^{42-45}$ (Figure 1).
Statistical analysis. Diagnostic accuracy was calculated for ELISA IgM to the single-plex PCR and TAC assays. Microscopic agglutination test was only performed on samples with a positive IgM ELISA reading, with specificity calculated for seroprevalence accuracy measurement.

Standard diagnostic accuracy indices of sensitivity, specificity with exact $95 \% \mathrm{Cls}$, as well as area under the receiver operator characteristic curves (AUROCCs) were calculated using Stata/IC 15.0 (StataCorp LP, College Station, TX). An area of $>0.8$ was considered good, $0.7-0.8$ was considered fair, 0.5-0.6 was considered poor, and $\leq 0.5$ was considered diagnostically uninformative. ${ }^{46-48}$

\section{RESULTS}

Blood samples were collected from children and adults in Thailand and Myanmar and their baseline characteristics are presented in Supplemental Table 1. Of the 740 blood samples collected, $656(88.6 \%)$ had a sufficient volume for the current analysis. The proportion of patients with a confirmed acute leptospirosis infection as defined by either single-plex or TAC PCR assay was 1.1\% (7/656), whereas 23.5\% (154/656) were seropositive by IgM ELISA. The AUROCC analysis was 0.65 (95\% Cl: 0.41-0.89) (Supplemental Table 2), suggesting the IgM ELISA to be diagnostically poor (Figure 2).

The IgM ELISA cutoff of 11 Panbio units (as per manufacturer's specifications) showed sensitivity and specificity of $71.4 \%$ and $36.4 \%$, respectively. Increasing the percentage of patients correctly classified to $>95 \%$ resulted in an improved specificity of $96.0 \%$ at a cutoff optical density (OD) of 1.56; however, the sensitivity was reduced to $14.3 \%$ (Supplemental Table 2).

Of the 154 positive IgM ELISA samples, all had a sufficient blood volume to be tested for the MAT. Of these, exposure as defined by MAT $\geq 1: 100$ was confirmed in 47 (30.5\%) samples, including $30(63.8 \%, 30 / 47)$ from Thailand and $17(36.2 \%$, $17 / 47)$ from Myanmar, and this difference was significant $(P$ value 0.030$)$. A titer of $\geq 1: 100$ was seen in 35 samples $(74.5 \%$, $35 / 47)$, 10 samples $(23.8 \%, 10 / 42)$ showed a titer $\geq 1: 200$, and two $(4.3 \%, 2 / 47)$ showed a titer of $\geq 1: 800$.

The specificity of IgM ELISA as a screening tool for recent exposure against MAT (defined as $\geq 1: 100$ ) was $39.9 \%$, using the manufacturer's recommendations. The highest specificity was measured at $98.0 \%$, using a cutoff OD of 1.73 .

\section{DISCUSSION}

We measured poor serodiagnostic performance from the Leptospira IgM ELISA compared with PCR assays on a single acute sample, considering outpatient children and adults attending primary care in Thailand and Myanmar. A similar finding was reported in Vietnamese clinics among non-severe patients; however, authors used an in-house ELISA as a reference method, challenging the interpretation of serodiagnostic accuracy. ${ }^{30}$ Similar performance has been reported in Lao PDR, with sensitivity of $95 \%$ and specificity of $41 \% .{ }^{29}$ However, samples originated from hospitalized and severe patients and relied on a suboptimal MAT dilution titer (i.e., $\geq 1: 400$ ). Other factors may explain such poor specificity for serodiagnosis, including the persistence of Leptospira lgM antibody for many months postrecovery, ${ }^{18}$ especially when PCR assays are used as a reference method. Nonetheless, cross-reactions to nonpathogenic Leptospira have been reported using the ELISA. ${ }^{30}$ 


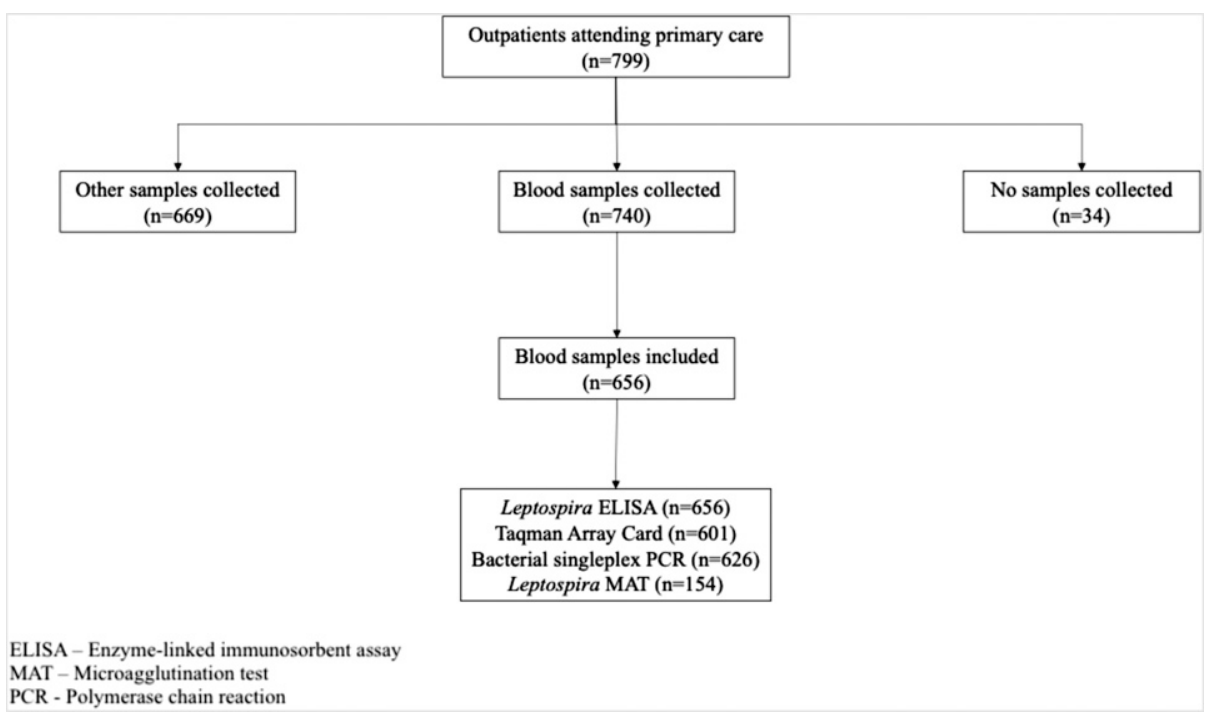

Figure 1. Diagnostic tests by sample type.

We also detected a substantial exposure in the community to pathogenic Leptospira using MAT (30.5\%). This indicates Leptospira to be an ubiquitous pathogen in Southeast Asia, which should be particularly highlighted in Myanmar where seroprevalence data were limited to animals before our study. ${ }^{12}$ There was a significant difference in exposure between Thailand and Myanmar, confirming the prevalence of Leptospira in rural environments. ${ }^{4}$

Using our seroprevalence data from the MAT, we evaluated whether IgM ELISA could be used as a screening tool for recent exposure to pathogenic Leptospira. ${ }^{33,40,49}$ Before our study, a single seroprevalence survey assessed ELISA accuracy against the MAT in Southeast Asia, reporting a specificity of around $93 \% .^{20}$ In this Malaysian study, the target population was healthy wet market workers and food handlers. Our lower specificity may be explained by differences in clinical status: serodiagnosis studies enrolling ill patients clinically suspected of leptospirosis similarly described lower specificities than in Malaysia. ${ }^{16,30,32}$ Adding low specificity measured in hospital-based studies to our own primary care-based

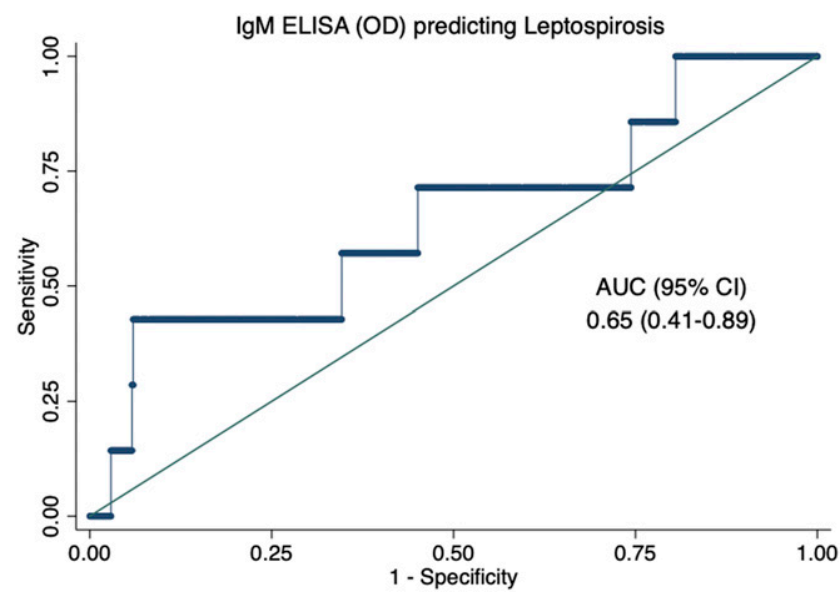

FIGURE 2. Area under the receiver operating characteristic curve of the Panbio Leptospira IgM ELISA vs. the PCR assay. evaluation, it is therefore unlikely that IgM ELISA may be useful for measuring a recent exposure to pathogenic Leptospira among febrile patients, regardless of the level of care.

Our study also suggests the ELISA diagnostic accuracy to be higher when the IgM cutoff is optimized using ROC curve analysis rather than based on the manufacturer's specifications. Further studies may aim to determine region-specific cutoffs in leptospirosis-endemic and non-endemic settings. However, our findings, combined with existing evidence, showed IgM ELISA to not be a promising candidate for diagnosing acute leptospirosis infection nor screening for recent leptospirosis exposure, regardless of the cutoff.

There were several limitations to our study. First, only acute samples were obtained, limiting the full serodiagnosis evaluation of Leptospira IgM ELISA. Second, the number of Leptospira spp.-confirmed cases using PCR assays was low, which may be attributed to the inclusion of non-severe patients with low bacterial loads, which lowered our detection sensitivity. ${ }^{50}$ Furthermore, the TAC assay, although highly specific, has shown low sensitivity in detecting bacterial pathogens. ${ }^{51,52}$ It has to be clarified that an IgM ELISA was used to test for seroprevalence and not lgG, whereas the MAT is composed of acute and chronic immunoglobulins. A peak in $\operatorname{lgM}$ is detected during early stages of infection and lasts for a few months, suggesting the measurement of a recent rather than past exposure. ${ }^{53}$ Finally, we did not test for MAT samples that were negative using IgM ELISA, and were therefore not able to calculate sensitivity.

\section{CONCLUSION}

The IgM ELISA, although simple and widely used, was found to be unsuitable for detecting acute leptospirosis infection among outpatient children and adults attending primary care, using PCR assays as a reference method. Its diagnostic accuracy may be improved by optimizing the cutoff based on a ROC curve instead of manufacturer's specifications. Even so, it is unlikely that such test, used on an acute sample, may represent an attractive candidate for the diagnosis of acute leptospirosis. Similarly, the IgM ELISA was not specific as a 
screening method for recent exposure to pathogenic Leptospira. Using the MAT, we demonstrated a significant exposure to pathogenic Leptospira among Thai and Myanmar communities, including rural and semi-urban settings.

Received July 8, 2020. Accepted for publication January 12, 2021.

Published online March 15, 2021.

Note: Supplemental tables appears at www.ajtmh.org.

Disclosure: S. D. B. and Yoel Lubell are supported by the Wellcome Trust of the United Kingdom.

Authors' addresses: Sandhya Dhawan, Mahidol-Oxford Tropical Research Medicine Unit, Faculty of Tropical Medicine, Mahidol University, Bangkok, Thailand, E-mail: sandhya@tropmedres.ac. Thomas Althaus and Yoel Lubell, Mahidol-Oxford Tropical Research Medicine Unit, Faculty of Tropical Medicine, Mahidol University, Bangkok, Thailand, and Nuffield Department of Medicine, Nuffield Department of Medicine Research Building, Centre for Tropical Medicine and Global Health, University of Oxford, Oxford, United Kingdom, E-mails: thomas.a@tropmedres.ac and yoel@tropmedres.ac. Duangjai Suwancharoen, Department of Livestock Development, National Institute of Animal Health, Ministry of Agriculture and Cooperatives, Thailand, E-mail: dj90705@hotmail.com. Stuart D. Blacksell, MahidolOxford Tropical Research Medicine Unit, Faculty of Tropical Medicine, Mahidol University, Bangkok, Thailand, Nuffield Department of Medicine, Nuffield Department of Medicine Research Building, Centre for Tropical Medicine and Global Health, University of Oxford, Oxford, United Kingdom, and Lao-Oxford-Mahosot Hospital-Wellcome Trust Research Unit, Microbiology Laboratory, Mahosot Hospital, Vientiane, Lao People's Democratic Republic, E-mail: stuart@ tropmedres.ac.

This is an open-access article distributed under the terms of the Creative Commons Attribution (CC-BY) License, which permits unrestricted use, distribution, and reproduction in any medium, provided the original author and source are credited.

\section{REFERENCES}

1. Fernando $N$, Wickremesinghe $S$, Niloofa $R$, Rodrigo $C$, Karunanayake L, de Silva HJ, Wickremesinghe AR, Premawansa S, Rajapakse S, Handunnetti SM, 2016. Protein carbonyl as a biomarker of oxidative stress in severe leptospirosis, and its usefulness in differentiating leptospirosis from dengue infections. PLoS One 11: e0156085.

2. Mullan S, Panwala TH, 2016. Polymerase chain reaction: an important tool for early diagnosis of leptospirosis cases. $J$ Clin Diagn Res 10: DC08-DC11.

3. Abela-Ridder B, Sikkema R, Hartskeerl RA, 2010. Estimating the burden of human leptospirosis. Int $J$ Antimicrob Agents 36 (Suppl 1): S5-S7.

4. Costa F, Hagan JE, Calcagno J, Kane M, Torgerson P, MartinezSilveira MS, Stein C, Abela-Ridder B, Ko Al, 2015. Global morbidity and mortality of leptospirosis: a systematic review. PLoS Negl Trop Dis 9: e0003898.

5. Torgerson PR, Hagan JE, Costa F, Calcagno J, Kane M, MartinezSilveira MS, Goris MG, Stein C, Ko Al, Abela-Ridder B, 2015. Global burden of leptospirosis: estimated in terms of disability adjusted life years. PLoS Negl Trop Dis 9: e0004122.

6. Pan American Health Organization, 2017. Leptospirosis - Fact Sheet. PAHO. Washington, DC: PAHO. Available at: https:// www.paho.org/hq/index.php?option=com_docman\&view= download\&category_slug=fact-sheets-4869\&alias $=39726$ leptospirosis-april-32017-39726\&ltemid=39270\&lang=en. Accessed May 5, 2020.

7. Agampodi SB, Dahanayaka NJ, Nockler K, Mayer-Scholl A, Vinetz $\mathrm{JM}, 2016$. Redefining gold standard testing for diagnosing leptospirosis: further evidence from a well-characterized, floodrelated outbreak in Sri Lanka. Am J Trop Med Hyg 95: 531-536.

8. Segura ER et al., 2005. Clinical spectrum of pulmonary involvement in leptospirosis in a region of endemicity, with quantification of leptospiral burden. Clin Infect Dis 40: 343-351.
9. Blasdell KR, Morand S, Perera D, Firth C, 2019. Association of rodent-borne Leptospira spp. with urban environments in Malaysian Borneo. PLoS Negl Trop Dis 13: e0007141.

10. Maciel EA, de Carvalho AL, Nascimento SF, de Matos RB, Gouveia EL, Reis MG, Ko Al, 2008. Household transmission of Leptospira infection in urban slum communities. PLoS Negl Trop Dis 2: e154.

11. Pappas G, Papadimitriou P, Siozopoulou V, Christou L, Akritidis $\mathrm{N}, 2008$. The globalization of leptospirosis: worldwide incidence trends. Int J Infect Dis 12: 351-357.

12. Victoriano AF et al., 2009. Leptospirosis in the Asia Pacific region. BMC Infect Dis 9: 147.

13. Sukmark T, Lumlertgul N, Peerapornratana S, Khositrangsikun K, Tungsanga K, Sitprija V, Srisawat N; Thai-Lepto AKI Study Group, 2018. Thai-Lepto-on-admission probability (THAlLEPTO) score as an early tool for initial diagnosis of leptospirosis: result from Thai-Lepto AKI Study Group. PLoS NegI Trop Dis 12: e0006319.

14. Ellis RD et al., 2006. Causes of fever in adults on the ThaiMyanmar border. Am J Trop Med Hyg 74: 108-113.

15. Blacksell SD et al., 2006. Limited diagnostic capacities of two commercial assays for the detection of Leptospira immunoglobulin $\mathrm{M}$ antibodies in Laos. Clin Vaccine Immunol 13: 1166-1169.

16. Niloofa $R$ et al., 2015. Diagnosis of leptospirosis: comparison between microscopic agglutination test, IgM-ELISA and IgM rapid immunochromatography test. PLoS One 10: e0129236.

17. Dutta TK, Christopher M, 2005. Leptospirosis-an overview. $J$ Assoc Physicians India 53: 545-551.

18. Cumberland P, Everard CO, Wheeler JG, Levett PN, 2001. Persistence of anti-leptospiral $\operatorname{lgM}$, IgG and agglutinating antibodies in patients presenting with acute febrile illness in Barbados 1979-1989. Eur J Epidemiol 17: 601-608.

19. Cumberland P, Everard CO, Levett PN, 1999. Assessment of the efficacy of an IgM-elisa and microscopic agglutination test (MAT) in the diagnosis of acute leptospirosis. Am J Trop Med Hyg 61: 731-734.

20. Samsudin S et al., 2018. Seroprevalence of leptospiral antibodies among market workers and food handlers in the central state of Malaysia. Trop Med Int Health 23: 327-333.

21. Gonwong S, Chuenchitra T, Khantapura P, Islam D, Ruamsap N, Swierczewski BE, Mason CJ, 2017. Nationwide seroprevalence of leptospirosis among young Thai men, 2007-2008. Am J Trop Med Hyg 97: 1682-1685.

22. Ahmed A, Engelberts MF, Boer KR, Ahmed N, Hartskeerl RA, 2009. Development and validation of a real-time PCR for detection of pathogenic Leptospira species in clinical materials. PLoS One 4: e7093.

23. Agampodi SB, Matthias MA, Moreno AC, Vinetz JM, 2012. Utility of quantitative polymerase chain reaction in leptospirosis diagnosis: association of level of leptospiremia and clinical manifestations in Sri Lanka. Clin Infect Dis 54: 1249-1255.

24. Liu J et al., 2016. Development of a TaqMan Array card for acutefebrile-illness outbreak investigation and surveillance of emerging pathogens, including ebola virus. J Clin Microbiol 54: 49-58.

25. Smythe LD, Smith IL, Smith GA, Dohnt MF, Symonds ML, Barnett LJ, McKay DB, 2002. A quantitative PCR (TaqMan) assay for pathogenic Leptospira spp. BMC Infect Dis 2: 13.

26. Silva MV, Camargo ED, Batista L, Vaz AJ, Brandão AP, Nakamura PM, Negrão JM, 1995. Behaviour of specific lgM, lgG and IgA class antibodies in human leptospirosis during the acute phase of the disease and during convalescence. J Trop Med Hyg 98: 268-272.

27. Limmathurotsakul $D$, Turner EL, Wuthiekanun $V$, Thaipadungpanit J, Suputtamongkol Y, Chierakul W, Smythe LD, Day NP, Cooper B, Peacock SJ, 2012. Fool's gold: why imperfect reference tests are undermining the evaluation of novel diagnostics: a reevaluation of 5 diagnostic tests for leptospirosis. Clin Infect Dis 55: 322-331.

28. Picardeau M, Bertherat E, Jancloes M, Skouloudis AN, Durski K, Hartskeerl RA, 2014. Rapid tests for diagnosis of leptospirosis: current tools and emerging technologies. Diagn Microbiol Infect Dis 78: 1-8. 
29. Tanganuchitcharnchai $A$, Smythe L, Dohnt $M$, Hartskeerl $R$, Vongsouvath M, Davong V, Lattana O, Newton PN, Blacksell SD, 2012. Evaluation of the standard diagnostics Leptospira IgM ELISA for diagnosis of acute leptospirosis in Lao PDR. Trans $R$ Soc Trop Med Hyg 106: 563-566.

30. Wagenaar JF, Falke TH, Nam NV, Binh TQ, Smits HL, Cobelens FG, de Vries PJ, 2004. Rapid serological assays for leptospirosis are of limited value in southern Vietnam. Ann Trop Med Parasitol 98: 843-850.

31. Reller ME, Bodinayake C, Nagahawatte A, Devasiri V, KodikaraArachichi W, Strouse JJ, Flom JE, Dumler JS, Woods CW, 2011. Leptospirosis as frequent cause of acute febrile illness in southern Sri Lanka. Emerg Infect Dis 17: 1678-1684.

32. Laras K et al., 2002. The importance of leptospirosis in southeast Asia. Am J Trop Med Hyg 67: 278-286.

33. Shafei MN, Sulong MR, Yaacob NA, Hassan $\mathrm{H}$, Wan Mohamad WMZ, Daud A, Ismail Z, Rusli Abdullah M, 2012. Seroprevalence of leptospirosis among town service workers in Northeastern state of Malaysia. Int $J$ Collab Res Intern Med Public Health 4: 395-403.

34. Rafizah AN et al., 2013. A hospital-based study on seroprevalence of leptospirosis among febrile cases in northeastern Malaysia. Int J Infect Dis 17: e394-e397.

35. Sundharagiati B, Harinasuta C, Photha U, 1966. Human leptospirosis in Thailand. Trans $R$ Soc Trop Med Hyg 60: 361-365.

36. Thai KT, Binh TQ, Giao PT, Phuong HL, Hung le Q, Van Nam N, Nga TT, Goris MG, de Vries PJ, 2006. Seroepidemiology of leptospirosis in southern Vietnamese children. Trop Med Int Health 11: 738-745.

37. Loong SK et al., 2018. Serological evidence of high Leptospira exposure among indigenous people (Orang Asli) in Peninsular Malaysia using a recombinant antigen-based ELISA. Trop Biomed 35: 1-9.

38. Althaus T et al., 2019. Effect of point-of-care C-reactive protein testing on antibiotic prescription in febrile patients attending primary care in Thailand and Myanmar: an open-label, randomised, controlled trial. Lancet Glob Health 7: e119-e131.

39. Ridzuan JM, Aziah BD, Zahiruddin WM, 2016. Study on seroprevalence and leptospiral antibody distribution among highrisk planters in Malaysia. Osong Public Health Res Perspect 7: 168-171.

40. Plank R, Dean D, 2000. Overview of the epidemiology, microbiology, and pathogenesis of Leptospira spp. in humans. Microbes Infect 2: 1265-1276.

41. Swapna RN, Tuteja U, Nair L, Sudarsana J, 2006. Seroprevalence of leptospirosis in high risk groups in Calicut, North Kerala, India. Indian J Med Microbiol 24: 349-352.
42. Jiang J, Chan TC, Temenak JJ, Dasch GA, Ching WM, Richards AL, 2004. Development of a quantitative real-time polymerase chain reaction assay specific for Orientia tsutsugamushi. Am J Trop Med Hyg 70: 351-356.

43. Henry KM, Jiang J, RozmajzI PJ, Azad AF, Macaluso KR, Richards AL, 2007. Development of quantitative real-time PCR assays to detect Rickettsia typhi and Rickettsia felis, the causative agents of murine typhus and flea-borne spotted fever. Mol Cellular Probes 21: 17-23.

44. Jiang J, Blair PJ, Felices V, Moron C, Cespedes M, Anaya E, Schoeler GB, Sumner JW, Olson JG, Richards AL, 2005. Phylogenetic analysis of a novel molecular isolate of spotted fever group Rickettsiae from northern Peru: candidatus Rickettsia andeanae. Ann New York Acad Sci 1063: 337-342.

45. Thaipadungpanit $\mathrm{J}$ et al., 2011. Diagnostic accuracy of real-time PCR assays targeting 16S rRNA and lipL32 genes for human leptospirosis in Thailand: a case-control study. PLoS One 6: e16236.

46. Hajian-Tilaki K, 2013. Receiver operating characteristic (ROC) curve analysis for medical diagnostic test evaluation. Caspian $\mathrm{J}$ Intern Med 4: 627-635.

47. Kumar R, Indrayan A, 2011. Receiver operating characteristic (ROC) curve for medical researchers. Indian Pediatr 48: 277-287.

48. Mandrekar JN, 2010. Receiver operating characteristic curve in diagnostic test assessment. J Thorac Oncol 5: 1315-1316.

49. Brockmann S et al., 2010. Outbreak of leptospirosis among triathlon participants in Germany, 2006. BMC Infect Dis 10: 91.

50. Reimer LG, Wilson ML, Weinstein MP, 1997. Update on detection of bacteremia and fungemia. Clin Microbiol Rev 10: 444-465.

51. Warhurst G et al., 2015. Diagnostic accuracy of SeptiFast multipathogen real-time PCR in the setting of suspected healthcareassociated bloodstream infection. Intensive Care Med 41: 86-93.

52. Weller SA, Cox V, Essex-Lopresti A, Hartley MG, Parsons TM, Rachwal PA, Stapleton HL, Lukaszewski RA, 2012. Evaluation of two multiplex real-time PCR screening capabilities for the detection of Bacillus anthracis, Francisella tularensis and Yersinia pestis in blood samples generated from murine infection models. J Med Microbiol 61: 1546-1555.

53. Lessa-Aquino $C$ et al., 2017. Distinct antibody responses of patients with mild and severe leptospirosis determined by whole proteome microarray analysis. PLoS Negl Trop Dis 11: e0005349. 